\title{
A new approach for using the hough transform to extract features from solar coronal images
}

\author{
Claudinei Walker da Silva ${ }^{1}$, Reinaldo R. Rosa ${ }^{1}$ and Joaquim E.R. Costa ${ }^{2}$ \\ Manuscript received on October 01, 2012 / accepted on December 21, 2012
}

\begin{abstract}
The study of the magnetic fields present in the solar corona is attracting increasing interest due to strong evidence of its relation to the phenomena observed in that region of the solar atmosphere. From the last two decades we have available images of the solar corona with increasingly better resolution, so that the comparison of these images with the physical models developed to study the dynamics of coronal magnetic fields is a task of extreme importance in solar astrophysics. We present in this work a new approach for the use of Hough Transform (HT) in order to extract features from the solar coronal images obtained in the Extreme Ultra-Violet (EUV). Our methodology is based on the fragmentation of the image and using of Hough Transform to get results from each fragment, and then identifying the fragments of the same magnetic field line using an interconnection algorithm. Finally we developed the evaluation of our methodology, where we proceed to compare the pixel coordinates of extracted magnetic field lines with those generated synthetically by the physical model. The features extraction algorithm is still under evaluation, but the early results have proved satisfactory.
\end{abstract}

Keywords: hough transform, features extraction, pattern recognition, computer vision, solar astrophysics.

\section{INTRODUCTION}

In general terms, the Sun is defined as an approximately spherical structure, where plasma is kept in such configuration by gravitational force. The solar region which is visible to the naked eye is called Photosphere, and layers above it constitute the solar atmosphere. Solar Corona is the outer region of the solar atmosphere, and it can be observed during total solar eclipses. A series of not yet understood phenomena occur in this region, such as solar radio bursts, solar flares and coronal mass ejections.

\subsection{The solar physics problem}

It is supposed that magnetic fields play a crucial role in the dynamics of solar corona and, therefore, study these structures is extremely important. These magnetic fields are still not observa- ble in all levels of solar atmosphere, and it was only in the last fifteen years that images from Extreme Ultra-Violet (EUV) and $X$-rays of Solar Corona have been obtained, evidencing the presence of magnetic force field lines [1]. It is known that force lines of coronal magnetic field are associated to the presence of sunspots observed in the Photosphere with magnetograms, which are a graphic representation of magnetic fields intensity variation. Upon the impossibility to observe magnetic field lines in all atmosphere levels, some physical models of magnetic fields have been developed. Virtually all of them try to reconstruct magnetic field lines from specially set of boundary conditions and the sunspots characteristics. These are known as magnetic field lines extrapolation methods, of which one of the most used is the Linear Force-Free Field (LFFF) extrapolation method [2], where it is considered that the plasma in the solar corona is mainly governed by

Correspondence to: Claudinei Walker da Silva-E-mail: claudinei.walker@gmail.com

${ }^{1}$ Laboratory for Computing and Applied Mathematics - National Institute for Space Research, São José dos Campos, SP, Brazil.

${ }^{2}$ Astrophysics Division, National Institute for Space Research - São José dos Campos, SP, Brazil. 
the action of magnetic field, leading the equation $\nabla \times \mathbf{B}=\alpha \mathbf{B}$, where $\mathbf{B}$ is the magnetic field and $\alpha$ is the proportionality term related to the current in the system. When $\alpha=0$ the field lines have a simple dipole configuration. The extrapolation methods result in an approximation of coronal magnetic field lines behavior and it is natural to compare the physical model with the observations. This task has long been carried out in a manual or semiassisted manner, which makes it inefficient and unpractical. There is no completely automatic method that recognizes and compares coronal magnetic field lines in EUV images with the field lines synthetically generated by extrapolation methods.

\subsection{Computer Vision in this context}

Computer Vision is a relatively new sub-area of Computer Science whose main objective is to extract numerical or symbolic information from images. "Computer Vision is the construction of explicit, meaningful descriptions of physical objects from images. Image understanding is very different from image processing, which studies image-to-image transformations, not explicit description building. Descriptions are a prerequisite for recognizing, manipulating, and thinking about objects" [3]. From this premise, the techniques and methods of Computer Vision have great importance in solution of a series of problems that involves the features extraction of images, which is exactly what we would do.

\subsection{Objective}

This work aims to develop an automatic methodology to recognize magnetic force field lines in solar coronal images obtained in EUV, extracting their features and comparing them to the ones synthetically generated by the physical model. For such purpose, a computational approach was adopted for the problem, focusing on processing coronal solar images, extracting the features of the field lines, calculating field lines from the physical model and finally comparing them to the ones generated by the model.

\subsection{Structure of this paper}

The section II presents the current methodologies to extract information from magnetic field lines from EUV images; section III presents our contribution to handle the features extraction problem, detailing the pre-processing, segmentation and information extraction steps; the section IV presents the evaluation of the methodology and shows the preliminar results of information extracted from field lines in images and the synthetically generated by the physical model, and finally on the section $\mathrm{V}$ we present our conclusions.

\section{CURRENT METHODOLOGIES}

The problem of recognition of the magnetic field lines has been handled in different ways in recent years, but there is no one fully automated method able to identify magnetic field lines in an image obtained in the EUV. Several initiatives are in progress, but in general the methods are not fully automated or recognize only a small part of the coronal magnetic structures. The proposed ideas generally seek to remove background noise to highlight the field lines and then use an approximation of magnetic field lines by a magnetic dipole, searching pixel-by-pixel of the brightest spots of the image.

\subsection{A method based on pixel connection}

The approach of the pixel-by-pixel search was used in a recent paper [4]. The EUV images of solar corona were preprocessed, removing the noise and enhancing the image sharpness through appropriate filters. Next, a segmentation algorithm was applied [5], generating a binary version of EUV image, highlighting the field lines, but increasing the remaining noise. From this step the authors used a simple dipole magnetic model to guide the pixel search, selecting the candidate pixel using some criteria of pixel connection to connect all candidate pixels of a magnetic field line. There is no comparison between this field line and that generated by physical model.

\subsection{A method based on active contours}

In the work of Gill, Fletcher \& Marshall in 2010 [6], where the objective was to monitor flares dynamics, active contours were used to extract information from a sequence of EUV images. To perform this, the authors used the original proposal of active contours [7] as a spline that changed for each step of processing, where an energy function was associated to this spline. This energy function was minimized until they reach a reference value previously established. The authors applied a pre-processing procedure on images using Solar Soft [8] computational framework. After the use of an edge detection algorithm, some control points were defined, using a distance of five pixels from each other. This set of control points is the initial active contour. Thereafter, using the minimization algorithm, the energy function for each control point was calculated, moving them to a position of lower energy. According to the authors, the method showed satisfactory results to images with low level of noise and well defined structures.

\section{A NEW APPROACH}

The approach proposed here arose from the need to develop a totally automatic recognition methodology for the coronal mag- 


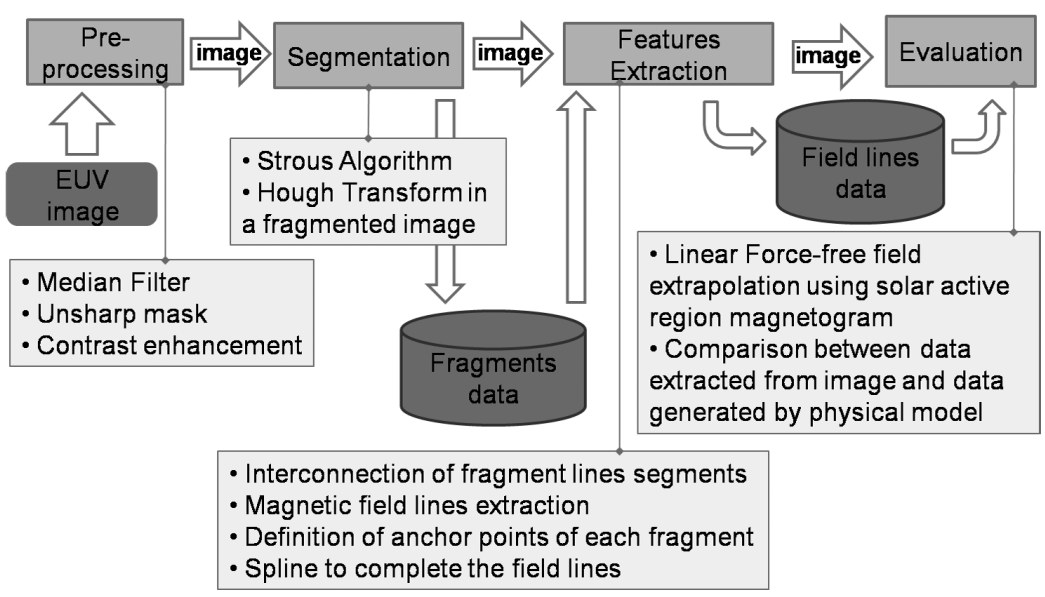

Figure 1 - The complete pipeline of our approach for the features extraction of magnetic field lines of EUV images.

netic field lines, considering the direction of these lines provided by EUV images. A literature search in this area has shown that this approach is unprecedented, since methods developed so far are semi-assisted, or only use information provided by EUV images for the recognition of magnetic field lines. Our approach is based on a combination of different computer vision techniques and uses the directional information of magnetic field lines. A diagram showing the pipeline of our approach can be seen in Figure 1. The first stage is a pre-processing, where part of noise is removed, the brightness and contrast are corrected, and the sharpness of image is enhanced. The second stage is the segmentation, where a gradient based algorithm is used to recover the brightest pixels of magnetic field lines. Next, we use a special approach of the Hough Transform, where the image is divided in fragments, and the Hough Transform is applied on each fragment, resulting in a set of data about all the fragments. In the next stage, we develop an algorithm that uses the directional information of magnetic field lines extracted from each fragment to connect a sequence of lines segments, recovering the complete field line. For each fragment, an anchor pixel is defined, and the coordinates of all anchor pixels are used to perform an interpolation. The information about all recovered lines was stored in database for later use in evaluation stage. This paper presents our approach to contribute to the solution of the problem, but the results are still being developed and are very preliminary.

\subsection{Pre-processing}

The images used in this paper were obtained by TRACE satellite in EUV, on spectral line $171 \AA$, as it is one of the lines with greater ionized iron emission intensity (Fe IX, on 171^) [9].
Image pre-processing basis consists on applying successive steps of noise reduction and image sharpness and contrast using different techniques. The first one, median filter, consists in correcting the intensity value of an image pixel based on the median of its adjacent pixel values. Taking as example a set of nine pixels arranged as a $3 \times 3$ matrix, the central pixel would have its intensity value substituted by the median of its eight adjacent pixels intensity values. In this development, a median filter is applied, with a $3 \times 3$ pixel mask, in order to minimize noise in the form of black and white pixels (known as salt and pepper noise) associated to highly energetic particles that reach instruments sensors. By using median filter, it is possible to guarantee that pixel intensity is kept in a level close to that of its adjacent pixels, considering an area of the size of the selected mask. Afterwards another pixel intensity correction is applied to image, aimed to enhance images sharpness. This proceeding is known as unsharp mask, where each pixels final intensity value is the result of the subtraction of a calculated intensity value from the original image pixels intensity value. This calculated intensity value is obtained by applying an average filter to the image resulting from the application of the median filter. The average filter operates as the same manner as the median filter described above, with an exception made to the calculation performed, which in this case is an average of the pixels intensity values. For the average filter, a $11 \times 11$ mask is used. Image sharpness is enhanced by taking the pixels from the field line structure. Finally, contrast is enhanced, generating a new image, whose pixels intensity values are obtained by calculating the square root of the sharpness-enhanced images pixel intensity value. The results of these successive steps can be observed in Figure 2. We found that the results obtained in the step of preprocessing depend on the values of the input parameters of 
algorithms, and these in turn depends on the input image, however, for the images of the solar corona with which we are working, after a few tests supported by the visual inspection, we get a set of values for the parameters which so far has shown satisfactory results. In part two of this paper we will discuss in depth the proper choice of these parameters.
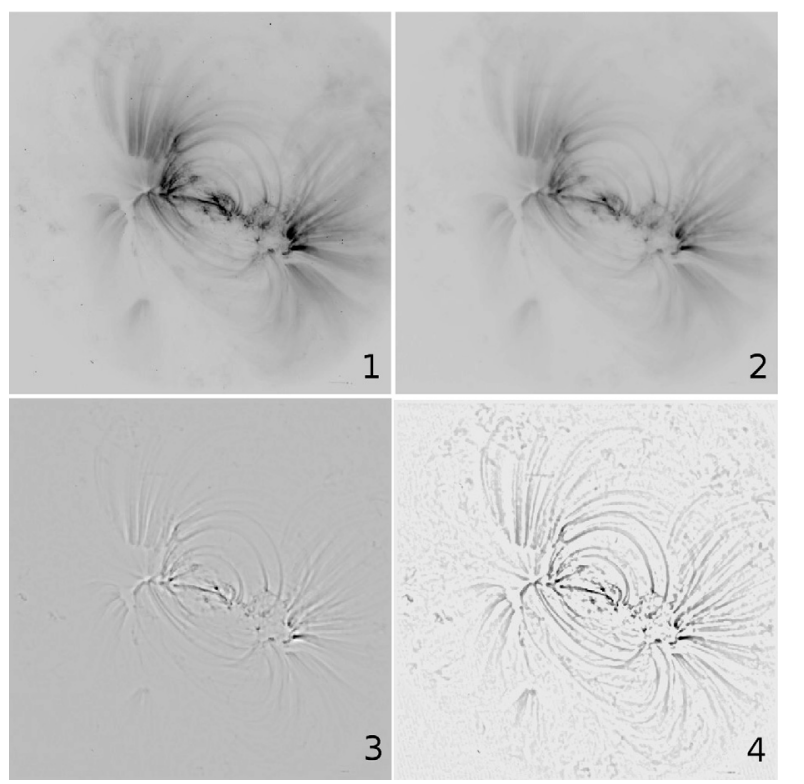

Figure 2 - Pre-processing of images obtained in EUV by TRACE instrument. (1) Original TRACE image, from May 22, 1998 22:21 UT. (2) Application of a median filter, with a $3 \times 3$ mask. (3) Application of an unsharp mask. (4) Contrast enhancement by use of a square root filter.

\subsection{Segmentation}

The aim in segmentation step is to highlight coronal magnetic field lines, in a way that segmented images could later be used in other analysis steps. Images used in this paper present very specific characteristics. Pixels being studied are usually distributed in elongated and narrow arc-shaped structures, so the use of classic segmentation algorithms, such as watershed and the ones based in edge detection are not the best solution for the images being worked on, as such algorithms do not provide the brightest regions of arcs with few pixels in width. The ideal would be using an algorithm which segmented lines and provided very narrow structures with width of one pixel.

Therefore, an attempt is made by using a segmentation algorithm that took into consideration the direction of the structures contained in the images. This study approach used Strous algorithm, which consists in a technique that highlights pixels based on their brightness gradient [5]. There are two variations of Strous algorithm, called 1 and 2. According to variation 1 , the pixel being processed is compared to its adjacent pairs, for each of the horizontal, vertical, and both diagonal directions. If this pixel is simultaneously larger than its vertical adjacent ones, for example, 1 is added in an accumulator of the same size of the original image. This is repeated for all directions. In the end of processing, these four matrices are added, so result matrix may contain values from zero to four. It is clear that structures with directional components tend to be highlighted, and will have significant values in the accumulator (3 or 4, for example). In algorithm 2, Strous changed the comparison: instead of testing if the pixel being processed is simultaneously larger than its adjacent ones, the average of both adjacent pixels is compared to the pixel being processed, in the indicated directions. Figure 3 presents how Strous algorithm works. Another interesting feature of Strous algorithm is the fact that it recovers the brightest part of the structure, instead of its edges, which occurs with edge detection algorithms. In a line of a certain width in pixels, if the brightest pixels are along the central part of the line, these will be the ones recovered.

Variation 1 of Strous algorithm is applied to each image obtained in pre-processing step (Fig. 2, images 2, 3 and 4). It is verified that the best results were obtained by using image 4 of Figure 2. This occurred because in this image coronal arcs are highlighted, with a significant noise reduction, comparing it to the original image (Fig. 2, image 1). These results can be seen in Figure 4.

An improvement of the segmentation obtained with Strous algorithm was developed by using a line extraction technique based on the fact that many magnetic force field lines present a curved-like and elongated geometrical structure. The idea was that, by dividing a curved line in small fragments, each of them becomes closer to being a straight line segment. Therefore, it is possible to use the Hough Transform [10] in this task, as it effectively recovers straight lines in noisy images. Hough Transform is based on a voting procedure, parameterizing the line equation and creating a discrete space of such parameters, called Hough space. For a line $\mathbf{a x}+\mathbf{b}$, Hough space would be formed by an accumulating matrix, whose columns would represent parameter a and whose rows represent parameter $\mathbf{b}$. Hough Transform algorithm goes over the image, pixel by pixel, and assesses if each of them falls on a line whose parameters are $\mathbf{a}$ and $\mathbf{b}$, in such case adding one vote to the element $[\mathbf{a}, \mathbf{b}]$ of the accumulator matrix. By the end of processing, there will be regions of Hough space with a greater counting for a specific pair $[\mathbf{a}, \mathbf{b}]$, so that a point in Hough space represents a line in the image space. 


\section{Strous Algorithm}
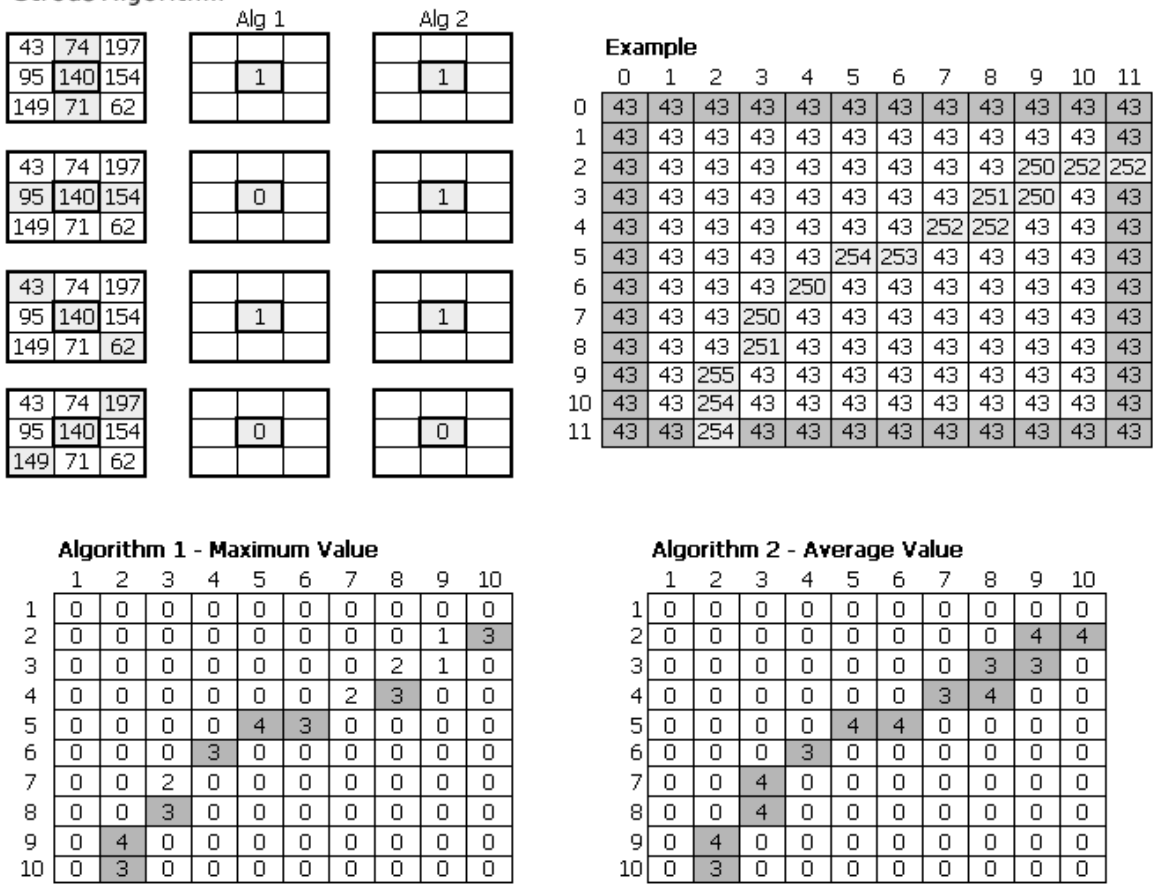

Figure 3 - Strous algorithm compares the processed pixel to its adjacent ones, considering four directions. Variation 1 of the algorithm considers the maximum value, while its variation 2 considers adjacent pixels average.

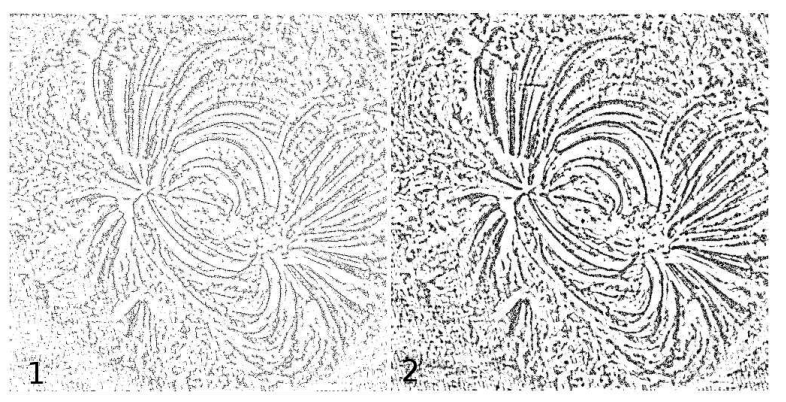

Figure 4 - Application of Strous Algorithm in image 4 of Figure 2. On the image 1 (left) we can observe variation 1 of Strous Algorithm, which takes into consideration pixels maximum value against its adjacent pixels. On image 2 (right), variation 2 considers central pixels value against adjacent pixels average. In this study algorithm 1 was used, as it results in images with thinner lines.

We develop an algorithm able to fragment an image and apply the Hough Transform on each fragment, and finally recompose the image. The image obtained from the initial segmentation, resulting from Strous algorithm is divided in same size fragments. For each fragment, Hough Transform is applied, storing the corresponding Hough space. In order to enable the separation of point clusters in Hough space, from k-means algorithm, which is much used in clustering analysis problems, it was necessary to develop an algorithm that returns the average coordinates of the point that best represents each point cluster of the line segment of that fragment. This way, it was possible to separate in Hough space the $\mathbf{a}$ and $\mathbf{b}$ coordinates representing the line segment of that fragment that presents the highest count, normally resulting in just one line in image space. In the event of more than one coordinates with same counting, the algorithm calculates the average of the coordinates, resulting in only one point in Hough space. It is possible due to typically small size of each fragment. This point in Hough space represents a line segment in image space. This procedure results in the mapping of all line segments of each image fragment, in local fragments coordinates (e.g.: $32 \times 32$ pixels fragment) and in image global coordinates (eg.: $1024 \times$ 1024 pixels image). By the end of processing, the algorithm rebuilds the entire image from each fragment. Fragment amount (and consequently their size) and pixel amount of each line segment are parameters of the algorithm. We develop a data structure to store all the data obtained in this stage, such as image metadata, as image size, fragments structure, amount of fragments, and fragments data, such as fragment identification, line segment parameters obtained from Hough Transform $([\theta, \rho]$ in polar coordinates, and $[\mathbf{a}, \mathbf{b}]$ in rectangular coordinates), local coordinates of line segment edges and local coordinates of central pixel of 


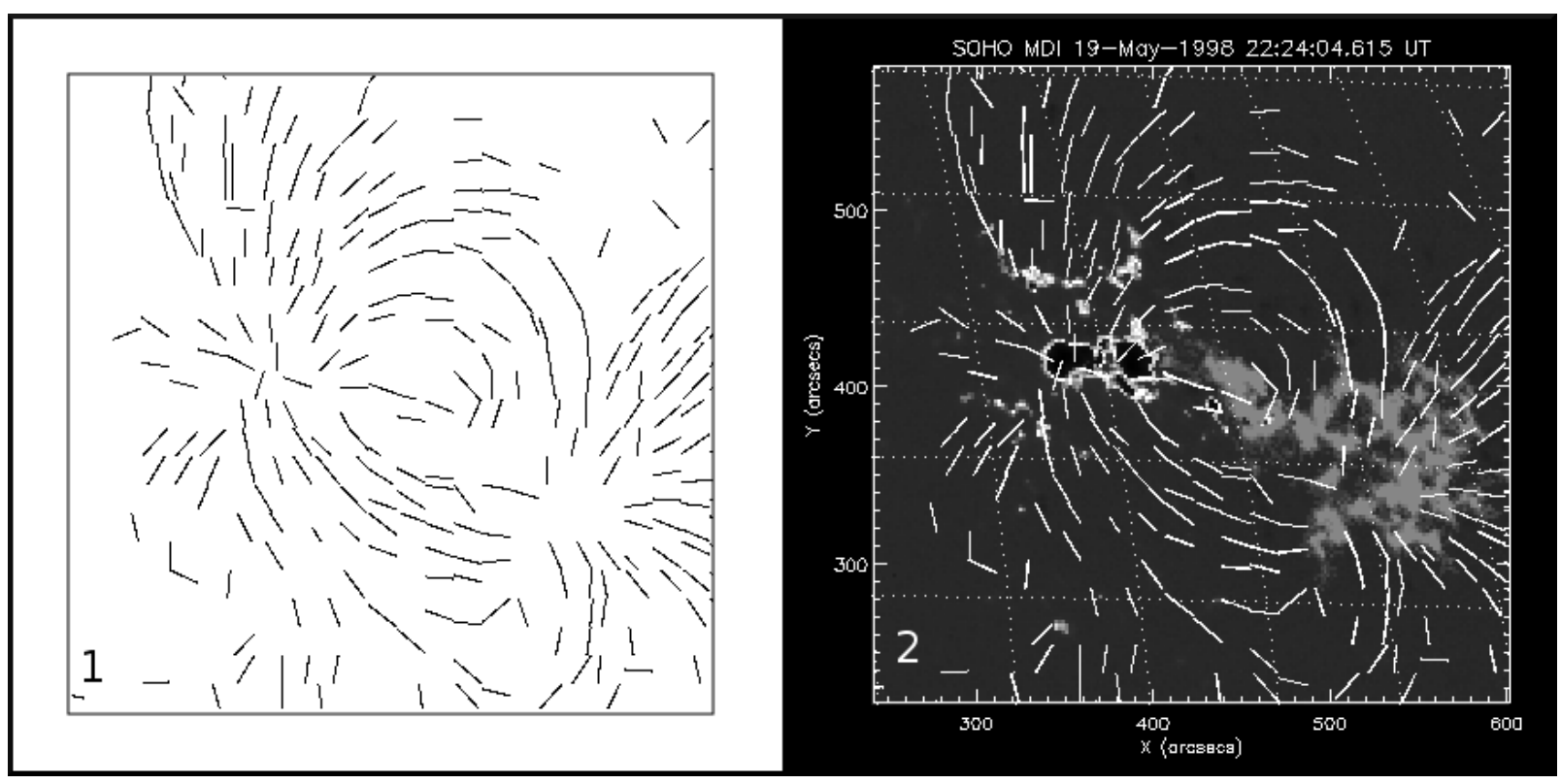

Figure $\mathbf{5}$ - The image 1 of Figure 4 is divided in 1024 fragments, and the algorithm of Hough Transform is applied in each one. The result is an enhance of segmentation, removing the noise of image, as shown in 1 (left). Data from entire image and each fragment are stored in specific database. Image 2 (right) shows the overlap of Hough Transform processed image with the magnetogram of solar active region. Is possible to verify the correspondence of magnetic sunspots on the base of magnetic arcs.

the line segment. Although k-means method presents known problems of convergence in some situations, this problem has minimal influence in our application, due to the fact that the data set in Hough space have low dispersion, since it is a fragment of the image with a size of $32 \times 32$ pixels where we usually find only one line segment, or a few line segments that represent the same magnetic fiel line. This particular feature of the data with which we are working minimizes the problem of convergence, but despite this, in part two of this paper alternative methods of grouping will be considered. Figure 5 shows the images obtained on the result of this stage.

\subsection{Features extraction of magnetic field lines}

Bibliography research provides several approaches used for recognizing magnetic field lines pattern in solar coronal images. Aschwanden et al. [11] presents a summary of semi-automatic methods developed in recent years. Such methods are able to recognize a small amount of arcs in very favorable conditions, differently from what can be found in reality. Most researched methods use a pixe/ search approach, based on criteria obtained in magnetogram and on the image obtained in EUV itself.

Our main goal in this work is to extract data with high semantic level of the EUV images and compare them with data generated from a physical model. We call this stage feature extraction, where we use data from fragments of an image obtained in the previous stage and develop an algorithm capable of sequencing fragments of a magnetic field line. For this we rely on the fact that each line segment in each fragment could be connected to another line segment of a neighbor fragment. If this occurred, the local coordinates of the pixels that are on the ends of line segments should have similar values. From this hypothesis, we developed an algorithm that for every fragment seeks in its 8-neighbor a fragment whose segments have similar coordinates of the coordinates of their line segment, which in effect would occur when the line segments constitute the same magnetic field line. This similarity criterion in fact corresponds to a distance from the ends of line segments in different fragments, measured in pixels in the $\mathbf{x}$ and $\mathbf{y}$ direction. This distance is a parameter of the algorithm. The Figure 6 shows the result of this stage of processing, highlighting the field line 123 composed by 7 fragments whose features were extracted from EUV image. The Table 1 shows the data of line 123, where the values of $\mathbf{a}$ and $\mathbf{b}$ are respectively the angular and linear parameter of the line segment in that fragment, in rectangular coordinates. The last four values are the local and global coordinates of the central pixel of the line segment.

The last step of feature extraction stage is an approximation by polynomial interpolation. The algorithm that makes the sequencing of the fragments also returns the local (for each fragment) and global (for the image) coordinates of the central pixel of each line segment. From these coordinates we have a sequence 
of pixels, which we call anchor points, from which we will apply a method of polynomial interpolation to finally get a continuous line that can be compared with that synthetically generated by the physical model. Taking for example the line 123 in Table 1, the columns Gcol and Grow shows the coordinates of the central pixel for each fragment. This step is under development, and we are studying what is the best interpolation method for our data. The first tests, not validated yet, are being conducted with the Piecewise Cubic Hermite Interpolating Polynomial (PCHIP) method.

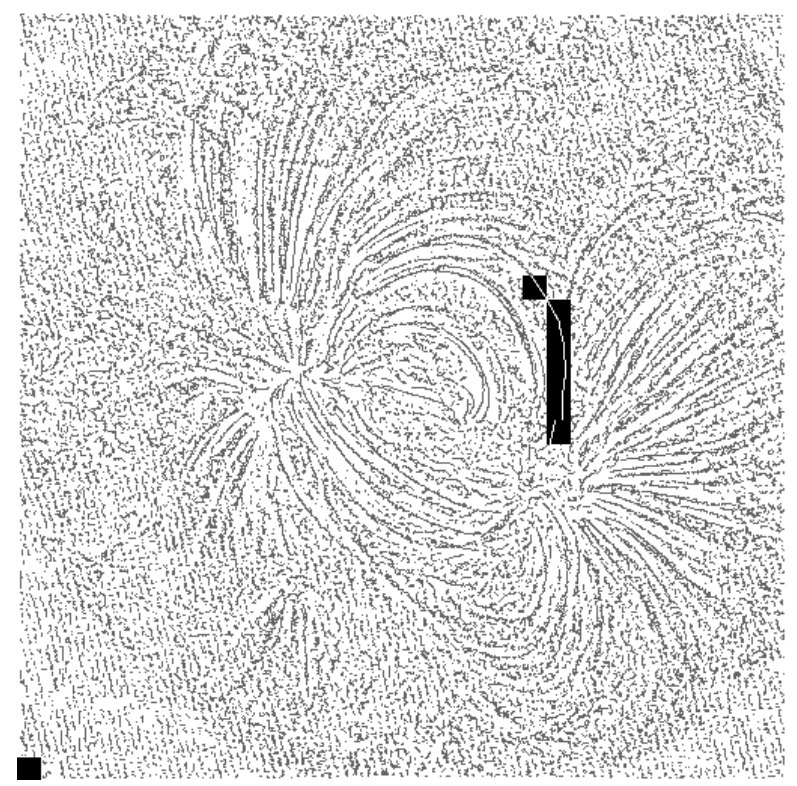

Figure 6 - The field line 123, which the data is show in Table 1. The small black square on left down corner represents approximately the size of each fragment in this example.

Table 1 - An algorithm for the features extraction of the field lines generates the data of field line 123 , formed by 7 fragments.

\begin{tabular}{c|ccccccc}
\hline Line & Frag & $\mathbf{a}$ & $\mathbf{b}$ & Lcol & Lrow & Gcol & Grow \\
\hline 123 & 470 & 4.32 & 35.45 & 7 & 16 & 711 & 464 \\
123 & 502 & 0.00 & 1.00 & 20 & 16 & 724 & 496 \\
123 & 534 & 7.28 & -51.41 & 23 & 16 & 727 & 528 \\
123 & 566 & -21.95 & 175.77 & 23 & 16 & 727 & 560 \\
123 & 598 & -4.32 & 17.72 & 19 & 16 & 723 & 592 \\
123 & 630 & -1.83 & -12.50 & 8 & 16 & 712 & 624 \\
123 & 661 & -1.49 & 8.96 & 21 & 16 & 693 & 656 \\
\hline
\end{tabular}

\section{COMPARISON AND VALIDATION}

The final stage of our methodology for the features extraction of the coronal solar magnetic field lines of EUV images consists of a comparison and validation procedure. The main idea here is to compare the data extracted from the images with those obtained by physical model. As already mentioned, one of the physical models adopted for the problem of extrapolation of magnetic fields in the solar corona can be the Linear Force-Free Field extrapolation method. Based on the methodology proposed by Nakagawa and Raadu [2], the solar physics group of the Astrophysics Division at National Institute for Space Research (in Brazil, INPE) has developed a code able to perform the extrapolation of the field lines, receiving the value of $\alpha$ in equation $\nabla \times \mathbf{B}=\alpha \mathbf{B}$ as input parameter. This code was used in a series of studies [12], and returns output vectors representing the three components $\mathbf{B}_{\mathbf{x}}, \mathbf{B}_{\mathbf{y}}$ and $\mathbf{B}_{\mathbf{z}}$ of magnetic field $\mathbf{B}$ in a spatial volume. From the positional parameters of the coronal EUV images, we will make the projection of the field $\mathbf{B}$ in the same direction of that image, obtaining a two dimensional view of the field $\mathbf{B}$, from where we will retrieve the position of the field lines generated by the model, and then we will compare these positions with those obtained by features extraction of the field lines in the images. Here we will use a method based on least squares fitting, or another that returns the distance between these two data sets. This procedure will be repeated for different extrapolated field lines, obtained with different values of $\alpha$, to verify the similarity of values.

\section{CONCLUDING REMARKS}

The main goal of this work is to develop a robust methodology for the features extraction of the coronal solar magnetic field lines. It is verified through image processing techniques that the application of successive filters is important to highlight the field lines, preparing image for a segmentation stage, which is developed by using Strous Algorithm, the division of image in a set of fragments and the use of Hough Transform in each fragment, rebuild the image at the end of processing. Segmentation results were satisfactory and compatible with the structures present in solar disc. For the features extraction stage, we develop a technique based on a connection of line segments of each fragment, and the determination of a set of anchor points, the central pixel of each line segment. These anchor points will be used to guide a polynomial interpolation method. Preliminary tests shows better results with the Piecewise Cubic Hermite Interpolation Polynomial (PCHIP) method, but not validated yet.

We will develop a validation procedure based on comparison of data extracted from EUV images with the data synthetically generated by LFFF method. To extrapolate the magnetic field lines we will use the code developed by astrophysics group of National Institute for Space Research (in Brazil, INPE), and 
compare, using a least square fitting procedure, the same two dimensional projection of synthetically field lines and the extracted field lines. This comparison procedure will be repeated for different values of $\alpha$ to check the better fitting.

Although we are still in final development phase of this work, we have already checked by visual inspection that the field lines extracted from the EUV images present a good fitting with magnetograms sunspots. We expect to complete shortly the proposed pipeline, and in the future we will present the final results of our work, in a part two of this paper, focusing on the validation approach.

\section{ACKNOWLEDGMENTS}

The authors would like to thank the Astrophysics Division and the Associated Laboratory of Computing and Applied Mathematics of National Institute for Space Research for the support to this research.

\section{REFERENCES}

[1] ASCHWANDEN MJ. 2005. Physics of the Solar Corona. An Introduction with Problems and Solutions ( $2^{\text {nd }}$ edition). SpringerPraxis Publishing.

[2] NAKAGAWA Y \& RAADU MA. 1972. On Practical Representation of Magnetic Field. Solar Physics, 25: 127-135.

[3] BALLARD DH \& BROWN CM. 1982. Computer Vision. PrenticeHall.
[4] LEE JK, NEWMAN TS \& GARY GA. 2006. Oriented connectivitybased method for segmenting solar loops. Pattern Recognition, 39: 246-259.

[5] STROUS LH. 2007. Loop Detection, Available in: $<$ www.Imsal.com/aschwanden/stereo/2000easton/cdaw.html>, acessed in 28/08/2007.

[6] GILL CD, FLETCHERL \& MARSHALL S. 2010. Using Active Contours for Semi-Automated Tracking of UV and EUV Solar Flare Ribbons. Solar Physics, 262: 355-371.

[7] KASS M, WITKIN A \& TERZOPOULOS D. 1988. Snakes: Active contour models. International Journal of Computer Vision, 1, 4: 321-331.

[8] BENTELY RD \& FREELAND SL. 1998. Crossroads for European Solar and Heliospheric Physics. Recent Achievements and Future Mission Possibilities. SOLARSOFT - an Analysis Environment for Solar Physics, ESA Special Publication.

[9] ASCHWANDEN MJ \& NIGHTINGALE RW. 2005. Elementary Loop Structures in the Solar Corona Analyzed from TRACE Triple-Filter Images. Astrophysical Journal, 633: 499-517.

[10] DUDA RO \& HART PE. 1972. Use of the Hough transformation to detect lines and curves in pictures. Commun. ACM, 15: 11-15.

[11] ASCHWANDEN MJ, LEE JK, GARY GA, SMITH M \& INHESTER B. 2008. Comparison of Five Numerical Codes for Automated Tracing of Coronal Loops. Solar Physics, 248: 359-377.

[12] SELHORST CL, COSTA JER \& SILVA AVR. 2005. 3-D Solar Atmospheric Model Over Active Regions, The Dynamic Sun: Challenges for Theory and Observations, ESA Special Publication. 